\title{
An Intersection based Traffic Monitoring using VANET
}

\author{
Shreya Sharma \\ M.Tech. CSE \\ Amity University \\ Noida, India
}

\author{
Manoj Kumar Sah \\ Assistant Professor \\ Department of CSE \\ NIT Jalandhar, India
}

\author{
Ashwani Malhotra \\ Assistant Professor \\ Department of CSE \\ NIT Jalandhar, India
}

\begin{abstract}
Due to the advent in technology and continuous progresses in wireless communication leads VANET as a research scenario which proposes communication between vehicles by wireless means. It has a large potential to improve safety and travel comfort of passenger. Developed areas suffer a major consideration for traffic safety as there is a high wastage of time and resources while traffic through traffic congestion. In this paper we have proposed a distance algorithm, which optimizes the traffic congestion problem. In this work we are using different simulation in which one is containing the application of isolator which determines the effect of normal traffic and other one is attack simulation which describes the effect of congested simulation network for the VANET. We have introduced Black hole attack at the intersection which leads congestion, and thus using distance algorithm. We removed the overcrowding over the circumstances using AODV routing protocol.
\end{abstract}

\section{Keywords}

Blackhole attack; Traffic congestion; Traffic monitoring; VANET; Distance Algorithm; AODV Routing Protocol; Vehicle to vehicle communication; vehicle to roadside communication.

\section{INTRODUCTION}

Traffic congestions are formed by many factors some is predictable like road construction, rush hour or bottleneck. Some traffic congestions are unpredictable for example human manners, weather and accidents. Drivers unaware of congestion eventually join it and increase the severity of it. The more severe the congestion is the additional time it will take to clear once the cause of it is eliminated [8]. The ability of a driver to know the traffic condition on the road ahead enables him or her to seek alternate routes for saving time and fuel [15]. When many drivers face traffic congestions that are explicitly those related to localized incidents such as accidents and temporary disruptions will be less severe and only the vehicle in the vicinity of the incident at the time of incident will be affected. Transition from the free flow to the congested traffic often occur at the highway spontaneously despite the fact that road could satisfy higher traffic demand. A congestion detection and dissemination system is being proposed using VANET that makes use of efficient communication channel, maintain location confidentiality and provide drivers about the real time information of traffic congestion in excess of long distances. The system uses vehicles itself prepared with simple inexpensive devices as the gatherer and distributer of the information excluding the need of costly road infrastructures such as sensors node, camera and external equipment. It focuses over the design and analysis of the key properties of the VANET and traffic control and monitoring enabled by the aforementioned technology. In this paper we have presented a strategy to reduce traffic congestion using vehicle to vehicle communication. Periodically emitted signals are used to analyze traffic and warn other drivers about possible occurrences of traffic break down. The proposed strategy is analyzed via computer simulations.

\section{GENERAL ARCHITECTURE OF VANET}

\subsection{Architecture of VANET}

The vehicular networking is fundamentally a wireless Ad-hoc network which provides communication between vehicles as
a. Vehicle to Vehicle communication
b. Vehicle to Infrastructure communication
c. Vehicle to Roadside Communication

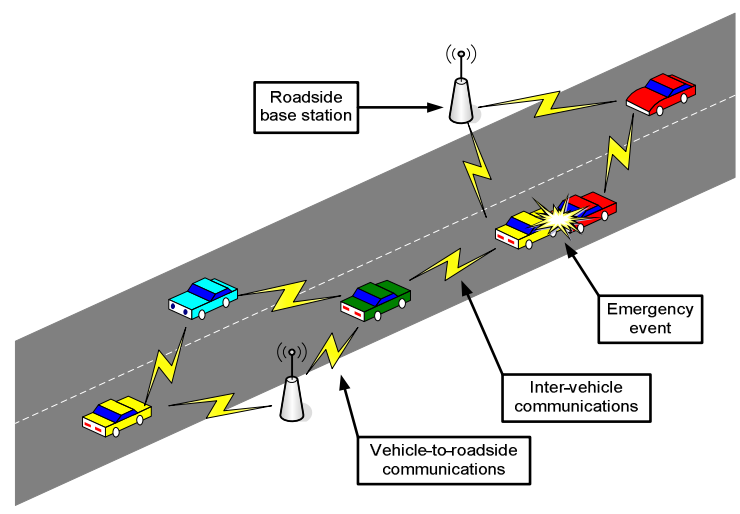

Fig. 1. General architecture of VANET

VANET consists of vehicles with on board sensors and road side units which provide communication from vehicle to vehicle or vehicle to infrastructure. Each vehicle communicates with one another in a dynamic Ad-hoc scenario. The traffic related information can be moved all around the network from the periodic beacons which may include safety driven alert application and infotainment including games.

\subsection{Factors influencing adoption of VANETs}

Here we have presented a variety of factor which influence the users for the implementation of VANETs in the present circumstances

a. Low Latency requirements for safety applications.

b. Safety applications like collision alert merge assistance, road situation warning etc requires messages to be propagated from the point of occurrence to the target vehicle within few seconds with least latency. In contrast to the $3 \mathrm{G}$ networks provide connectivity but have latency issues. 
c. Extensive growth of the multimedia and interactive applications.

d. Many applications such as gaming require continuous internet connectivity for such we need to have a vehicle to infrastructure connectivity domains and VANET supports such features.

e. Increasing concerns about privacy and security

f. Although Vehicle to Vehicle (V2V) communication may lead varietal security problem thus presence of Vehicle to Infrastructure (V2I) communication provide an efficient mechanism through centralized agencies can selectively broadcast the temporary identities with the public keys in the selective geographical area for the vehicles or the vehicles can pick up their required public keys in contrast to $\mathrm{V} 2 \mathrm{~V}$ where there occurs the problem with public key authentication.

\subsection{Current solutions to traffic congestion}

Most of the current navigation systems are static. Traffic decisions are not provided dynamically. In static system route selection is based on static map data, which does not provide an efficient route to driver. Most recently some devices have incorporated real time traffic information systems to aid in route selection.

This may include real time systems like NAVTEQ [5], [6] which rely on human or any road infrastructure like traffic camera and radar in order to maintain a central database for traffic conditions [13]. Thus limits its reach ability to major areas and reduces its utility.

In New York, 81 cameras were placed which can provide only visual information. These needs to be translated into congestion information either by humans or any specialized software. This was only one way oriented where information is received either by FM radio or satellite.

For the sake that vehicles could transmit data in real time to central infrastructure it is required that enormous communication infrastructure must be established. In two way communications such as On star [2] they rely on cell phone communication which may not be always switch on or within range and may not be designed for huge volume of communication as required by the traffic traveling transversely. Thus for this purpose we have proposed a technique where distributed approach is being utilized which uses vehicle themselves as a node in vehicular Ad-hoc network as well as a data gathering devices that collect the necessary information to determine current traffic conditions and disseminate them over long distances using V2V communication as well as such information into the vehicles navigation may provide driver with aware traffic routing using an efficient routing algorithm that takes into account traffic information. It is the most efficient approach as it provides a greater degree of flexibility and reduces the investments as cost is distributed among many drivers.

\section{RELATED WORKS}

Most of the research work in VANET focuses on simulating the vehicular traffic and multi-Hop routing. To discover and disseminate traffic congestion problem in VANET is a research issues [8]. Vehicle based GPS systems can be used in order to search and disseminate the traffic congestion information.

\subsection{Review on Some Existing works}

Collision avoidance systems [7], [9] are designed which detect traffic incident in real time and convey such information to the nearby vehicles in order to avoid accident. But these systems were different from the traffic congestion systems as the information was to be conveyed over short distances and needs to be extremely reliable while in later the information is to be relayed remains current and thus need to be disseminated and used for congestion amelioration.In recent year several researchers are working on the issue related to distributed detection and dissemination of the information.

Folkmoot et al [4] proposed a system which uses GPS systems for disseminating the traffic information. They called these systems as COC for VANET. This basically contains three information as raw information (level1), Density information (level 2) and congestion area information (level 3) and higher levels may contain aggregated information.

Onrush et al [3] from university of Maryland proposed a Street Smart system for congestion detection that uses clustering as a data aggregation technique to combine related recording of an unusually slow speed. Street smart uses clustering algorithm that works over a distributed network where each node analyses the collected statistics thus eliminating the need of central entity. The grouping of data points together which are similar in some aspects is called Clustering of information.

Yoon, Nobel and Liu of university of Michigan [10] proposed a traffic estimation system based on road segmentation and complexity of inner traffic scenarios. Some of these systems rely explicitly or implicitly on receiving the location from the vehicles in order to detect the congestion. When the size of congestion exceeds a particular transmission range which generally occurs in expressway scenario, the multi-Hop communication come into existence which concludes a problem whenever we want to keep location information anonymous a vehicle may receive fresh information and rebroadcasted information from the same vehicle. Since vehicles move frequently, these two sections of information will be identical. Hence systems rely on unique IDs as a method to identify source of each section of information and maintain a unique location for each vehicle. The broadcasting of unique vehicle IDs at the application level facilitates location tracking and thus leading privacy concerns. Recently many organizations in US have realized the importance of vehicular communication.

Dash Navigation, Inc [1], [2]; a start up in sunny valley in 2009 provided services to allow drivers to broadcast their speed and location in exchange of receiving updated traffic information compiled from other drivers in the network. This is a centralized system and relies on wireless internet connectivity which is not globally available everywhere as the central authority concerning is trusted location and privacy concerns are thus mitigate. Traffic view concerns with the estimation of the overcrowding on road.

\subsection{VANET}

Vehicular Adhoc network is a technology that uses moving nodes in the network to create a mobile network. VANET turns every participating node into a wireless router or node allowing vehicles to connect one another. If a vehicles drop out of a scrupulous range other vehicles can join in relating vehicles to one another so that a mobile internet is created. 
VANET is basically a subgroup of MANET where nodes refer to a vehicle network. VANET turns each participating node into a wireless router or node allowing vehicles to connect one another [14]. If one vehicles drop out of a particular range other vehicles can connect in linking vehicles to one another such that a mobile internet is created. VANET is basically a subgroup of MANET where nodes refer to a vehicle.

\section{PROPOSED WORK}

VANET has been studied in many fields since it has the ability to provide a variety of services such as detecting oncoming collision and providing warning signal alerts to the driver.

The services provided by VANET are often based on collaboration among vehicles that are equipped with relatively simple motion sensors and gap units. It has been proposed as an automated solution too many troubles allied to transportation, driving assistance and highway protection. VANET make possible by providing a communication among vehicles. Our main goal to build a communication scenario, depict vehicle to infrastructure and vehicle to vehicle communication.

\subsection{Packet loss due to black hole}

There can be situations when the vehicles receiving the signal may or may not respond the receipt of signal because of increased packet density leading more congestion as they may be exceeding the particular distance range or out of range. There may be packet loss because of certain network problem leading black hole

In order to overcome such problem we have proposed a distance algorithm. Here in this algorithm we have proposed a distance major which eliminates the congestion by providing a fixed distance constraint for the information exchange between the vehicle and the signal, thereby using vehicle to infrastructure communication.

The distance being fixed between the vehicle and signal is Euclidian distance. Signal information is being send or received only after the vehicle reaches the Euclidean distance which is being measured for nodes in $\mathrm{x}$ and $\mathrm{y}$ planes, where nodes serve as position of a vehicle. With distance major we can bring about the time required to gain sense of vehicular direction movement from traffic signal. Suppose that we have placed a clock at indicator designed for 5 minutes and then the vehicles incoming at the distance of communication may receive the information and will wait till the signal change from red to green, allow the vehicles in the correspondent location to move.

\subsection{Deployment of sensor nodes}

In this paper we are working over VANET network. We deployed the sensor network as per requisite simulation of VANET structure in which there are four roads preceding the traffic pattern through the signal of traffic go lumber or circle. Moreover, we are using eight vehicles on the road two in each road.

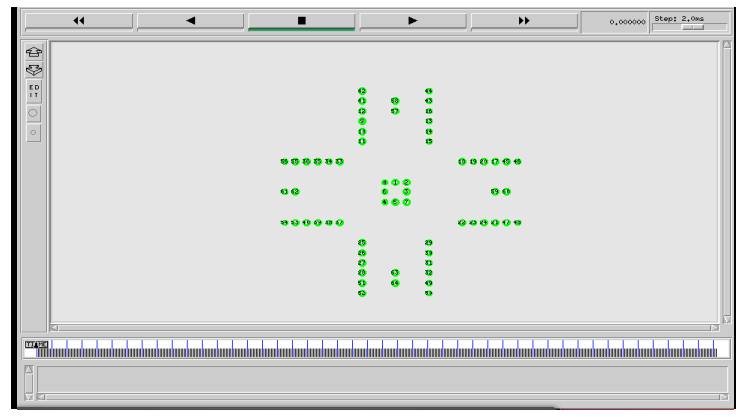

Fig. 2. Intersection simulation showing of 8 vehicles as node over four respective corners of traffic signals

As per requisite work we have to compare the effect of normal traffic and congested traffic. Thus we are using different simulation one is containing the application of isolator which determines the effect of normal traffic and other one is attack simulation which describes the effect of congested simulation network for the VANET.

At first we are showing the normal traffic pattern in which initially the first vehicle start moving from their points. If it receives yellow signal then it comes in distinct network range or distance and after getting the green signal starts communicating to the traffic control nodes and then further moves to their destination. The rest of the three vehicles also maintain the traffic signal pattern and accurate timing patterns for which the vehicles start moving only when they desired for the movements as per traffic rule and crossing of the last vehicle from their range. Similarly the rest of all vehicles follow the same procedure without any congestion.

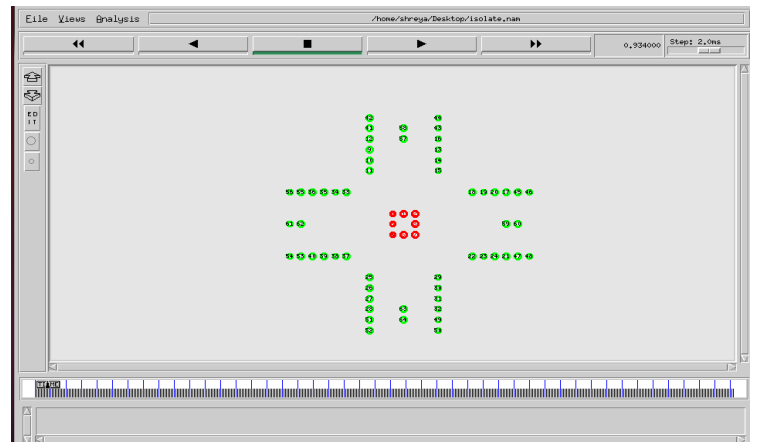

Fig. 3. the simulation begins at 0.8 seconds displaying red signal

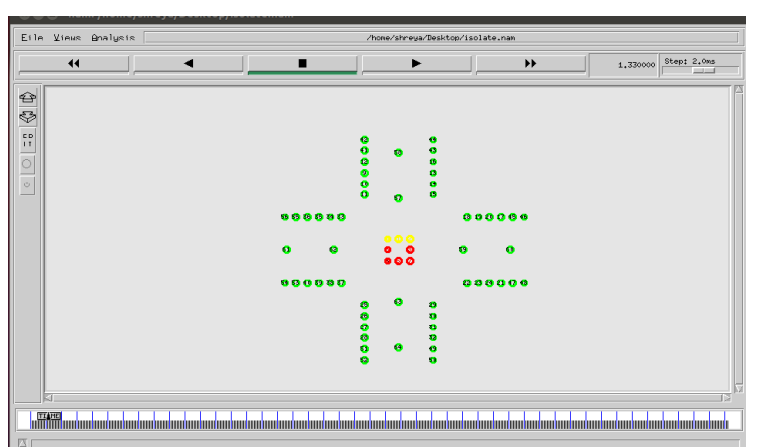

Fig. 4. The vehicle comes in the particular network range displaying yellow signal 


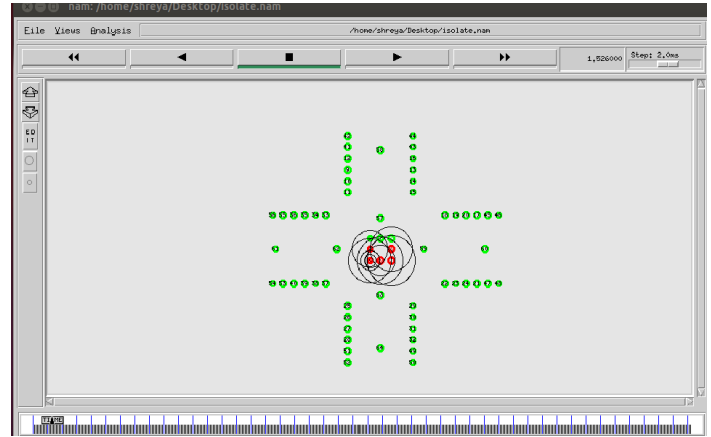

Fig. 5. The V-I communication at a particular distance and thus green signal, allowing vehicles to move to their destination

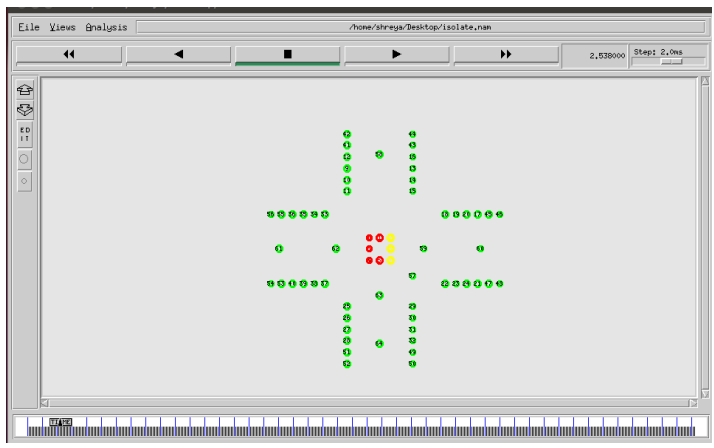

Fig. 6. The adjacent vehicle start communicating

Again, we are using the same simulation model as per isolator, the only difference is that we have implemented attack in place of isolator and there is no distance description in this scheme. Here a black hole attack is being introduced which disables the communication among the vehicles.

So due to the effect of attack whenever the nodes have to communicate or further move to their destination, they (four starting vehicle) remain blocked. They do not communicate and there are no movements due to the effect of attack.

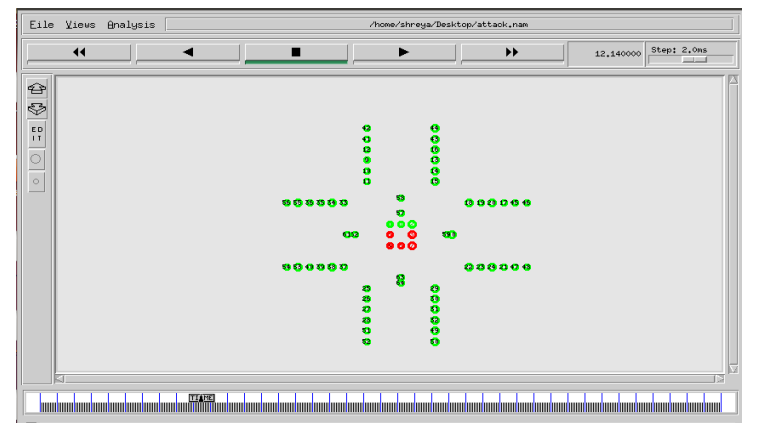

Fig. 7. A black hole attack leading communication failure leading congestion

After this, the rest of the four vehicles which are coming from backwards collide to the prior vehicle which clearly depicts the concepts of congestion and further move as per traffic control by demonstrating the effect of congestion and they move normally as per traffic situation.

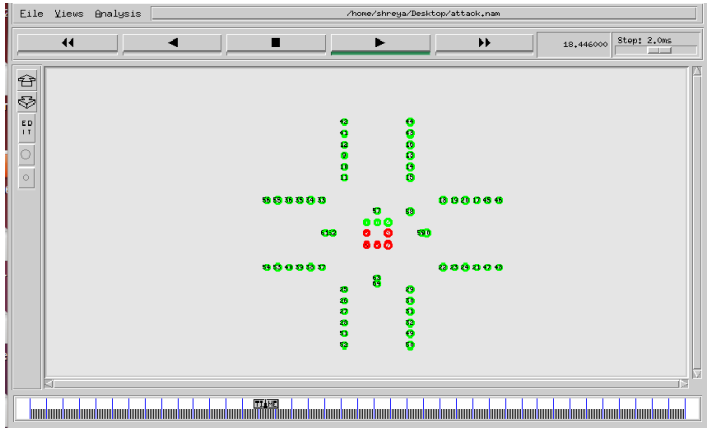

Fig. 8. The congestion and vehicular movement

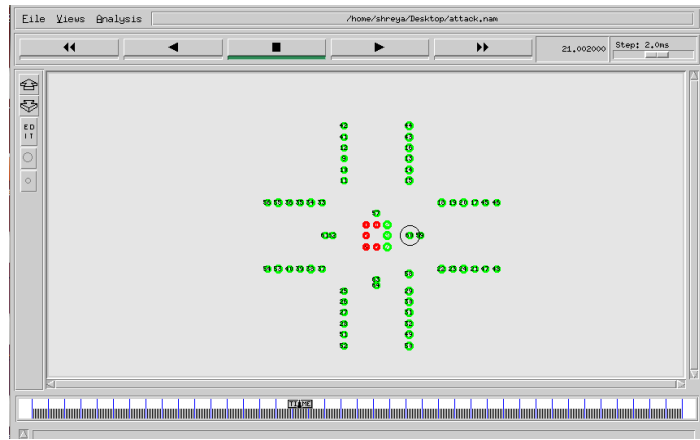

Fig. 9. Vehicular overtaking occur as the initials stop communication because of attack

\subsection{Calculation of Euclidean distance}

The distance algorithm is given for a vehicle is as follows. Consider for any two vehicles treated as nodes $\mathrm{P}$ and $\mathrm{Q}$

Step 1: The nodes $\mathrm{P}$ and $\mathrm{Q}$ is described by the coordinates $\left(\mathrm{p}_{1}, \mathrm{p}_{2}\right)$ and $\left(\mathrm{q}_{1}, \mathrm{q}_{2}\right)$

Step 2: Find the distance between the two nodes as $\left|\mathrm{p}_{1}-\mathrm{q}_{1}\right|$ and $\left|\mathrm{p}_{2}-\mathrm{q}_{2}\right|$

Step 3: Euclidean distance is thus given by applying Pythagoras over the above distances

Step 4: The distance between the two nodes $P\left(p_{1}, p_{2}\right)$ and $\mathrm{Q}\left(\mathrm{q}_{1}, \mathrm{q}_{2}\right)$ is $\sqrt{\left(p_{1}-q_{1}\right)^{2}-\left(p_{2}-q_{2}\right)^{2}}$

Step 5: Similarly for $\mathrm{n}$ number of nodes may be given as

$$
\sqrt{p_{1}{ }^{2}+p_{2}{ }^{2}+p_{3}{ }^{2}+\ldots \ldots+p_{n}{ }^{2}}
$$

\subsection{Proposed Algorithm}

A. For Isolate Scenario

For $\mathrm{i}=\mathrm{N}$ down to 0

do

begin

Step 1: Assign $\mathrm{v}_{1}, \ldots \ldots, \mathrm{v}_{\mathrm{n}}$ to isolate scenario.

Step 2: Simulation start at $t=0.8$

Step 3: Nodes $\mathrm{v}_{1}, \ldots, \mathrm{v}_{\mathrm{n}}$ active for communication.

Step 4 : for $\mathrm{i}=1$ to $\mathrm{n}$ do
a. $\mathrm{d}=$ infinity
b. $\mathrm{dv}_{1}=\mathrm{dv}_{2}$, black hole

Step 5: Congestion detection, break and return

B. For Attack Scenario

For $\mathrm{i}=\mathrm{N}$ down to 0 do

Assign $\mathrm{v}_{1} \ldots \ldots, \mathrm{v}_{\mathrm{n}}$ to attack scenario

begin

Step 1: Simulation start at $\mathrm{t}=0.8$

Step 2: Nodes $v_{1}, \ldots, v_{n}$ active for communication 
Step 3 : Calculate Euclidian distance $\mathrm{v}_{1}, \mathrm{v}_{2}$ as $\left|\mathrm{p}_{1}-\mathrm{q}_{1}\right|$ and $\left|\mathrm{p}_{2}-\mathrm{q}_{2}\right|$

Step 4 : for $\mathrm{i}=1$ to $\mathrm{n}$

do

$$
\begin{array}{ll}
\text { a. } & \mathrm{d}=\sqrt{\left(p_{1}-q_{1}\right)^{2}-\left(p_{2}-q_{2}\right)^{2}} \\
\text { b. } & \text { eud }=\mathrm{d} \\
\text { c. } & \mathrm{dv}_{\mathrm{i}}=\text { eud from traffic signal } \\
\text { d. } & \text { V-I communication } \\
\text { e. } & \text { Return and move to next zone } \\
\text { f. } & \text { end }
\end{array}
$$

Step 5 : Congestion free V-I Communication

Step $6:$ Repeat till $\mathrm{N}=0$

Step 7 : Break and Return

In this algorithm

$\mathrm{N}=$ Number of vehicular nodes

$\mathrm{v}_{1}, \mathrm{v}_{2}=$ vehicular nodes

$\mathrm{d}=$ distance between $\mathrm{V}$-I

eud = Euclidian distance between V-I as calculated between $(\mathrm{x}, \mathrm{y})$ coordinates. Let $\left(\mathrm{p}_{1}, \mathrm{q}_{1}\right)$ and $\left(\mathrm{p}_{2}, \mathrm{q}_{2}\right)$ is $(\mathrm{x}, \mathrm{y})$ coordinates for any vehicular node $\mathrm{v}_{1}$.

\section{SIMULATION RESULTS}

In this paper we have analyzed the isolate and attack traffic scenario based on the throughput and overhead Throughput describes the rate of successful message delivery over the network, the below graph represents the delivered throughput comparison in both the scenarios.

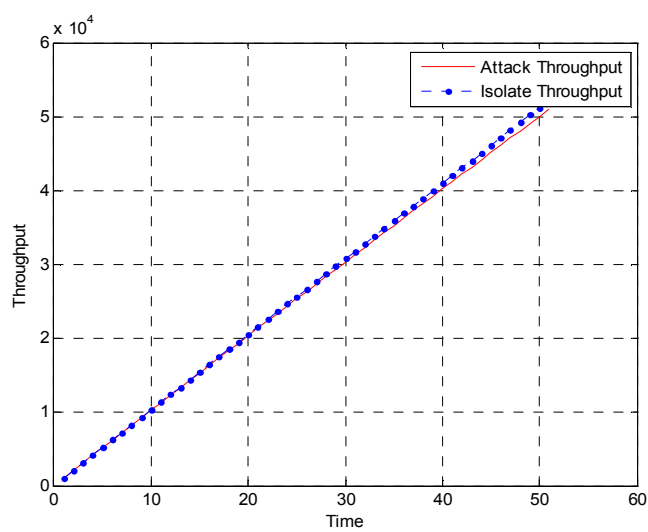

Fig. 10. Comparision of Throughput (Throughput for with and without Attack)

Overhead is the main reasons which may lead congestion and any scenario having congestion have maximum overhead

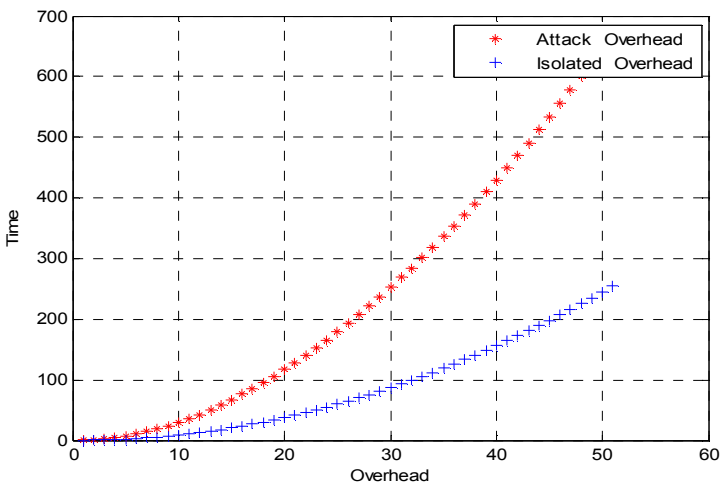

Fig. 11. Overhead Comparison

\subsection{Advantages of Proposed Algorithm}

The key advantages of the proposed system is that good network mainly concerns with efficient data transfer ,cost reduction, decreased packet loss, increased throughput and reduction in network overload. VANET systems offer certain characteristics they are:

a. Vehicles able to move at high speed

b. It is having a predictable mobility pattern as movement is constraint to road infrastructures.

c. VANET assume a large coverage area and thus are most convenient for the vehicles providing range over big distances.

d. Vehicles itself produce power, power consumption is not a major concern.

e. Vehicles already have a high cost thus embedding few sensors might not impact much over the overall expenditures.

f. VANET topology is very dynamic as vehicles come into and come out of vehicular transmission range with high speed.

g. Vehicles travel over long distances in a very little time in comparison to other mobile network

\section{CONCLUSION}

In this paper we have proposed a distance algorithm. We have also successfully reviewed various existing approaches. To remove traffic congestion we have implemented a solution which avoid packet loss occurring due to highly crowded vehicular traffic which get accumulated at an intersection may cause miscommunication due to signal loss . In our distance algorithm the communication occurs only when vehicle reaches a Euclidean distance from the traffic signal and there receive the information for the vehicular movement. For routing we have used routing protocol as ADOV.

We have implemented two major scenarios. First is isolated and second is attack traffic scenario where distance or network range is the constraint is defined over which we performed analysis in terms of throughput and overhead. We have described simulation results and a comparative analysis of throughput and overhead over network.

The particular approach can be represented as distributed computing paradigm that assumes vehicles as nodes in a vehicular Adhoc network as well as data gathering devices are preserved to collect essential information in order to determine current traffic conditions. Thus disseminate it over long distances using V2V communication. This distributed approach provides a greater degree of reliability and flexibility also it does not require a great investment over infrastructure as cost is being distributed among many vehicles acting as devices.

\section{REFERENCES}

[1] Car2Carcommunication consortium. www.car2car.org [Online].

[2] Dash Express: The first two-way, Internet-Connected GPS Navigation system. [Online]. www.dash.net.

[3] Dornbush, S. and Joshi, "Street Smart Traffic: Discovering and Disseminating Automobile Congestion Using VANET's" ISSN :1550-2252, IEEE (2007)

[4] Folkmoot, junya, etal Analytic, "Method for Real time traffic problems by using Content oriented communication in VANET", $7^{\text {th }}$ international conference on ITS, pp.1-6 (2007) 
[5] NAVTEQ Real time Traffic service [online] www.navteq.com.

[6] Chowdhury Sayeed Hyder, Anindya Iqbal: Towards Automated Traffic System Using Vehicular Network with Directional Antenna. FGCN, IEEE, pp. 454-458 (2008)

[7] Rawashdeh, Z.Yand Mahmud, S.M.Intersection Collision avoidance System Architecture System architecture. $5^{\text {th }}$ IEEE Consumer Communication and Networking Conference, pp.493-494 (2008)

[8] Scharank, David and Lomax, Tim :The Urban mobility report Technical Report s.1.: Texas Transportation Institute (2007)

[9] VANETwikipedia.org[online]en.wikipedia.org/wiki/

[10] Une Thoing Rosi, Chowdhury Sayeed Hyder, Tai-Hoon Kim: A Novel Approach for Infrastructure Deployment for VANET. FGCN, IEEE: pp. 234-238 (2008)

[11] Fabio Ricciato, Marco Mellia, Ernst W. Biersack: Traffic Monitoring and Analysis. Springer ( 2010)

[12] Richard Gilles Engoulou, Martine Bellaïche, Samuel Pierre, Alejandro Quintero: VANET security surveys. Computer Communications 44: 1-13 (2014)

[13] T. Nadeem, S. Dashtinezhad, "Traffic data dissemination using car-to-car communication", ACM Sigmobile Mobile Computing and Communications(2004)
[14] Kitani, Grad: Efficient VANET-Based Traffic Information Sharing using Buses on Regular Routes IEEE pp. 3031 - 3036 (2008)

[15] Jungkeun Yoon, Brian Noble, Mingyan Liu:Surface street traffic estimation MobiSys. IEEE pp. 220-232 (2007)

[16] Liren Zhang, Hesham El-Sayed, Valeriu Beiu, "A Position-Based Broadcast Relay Approach in Mobile Vehicle-to-Vehicle Network" , ICWN 2010: 611-616 ( 2010)

[17] Liren Zhang, Hesham El-Sayed, "A Novel Cluster-Based Protocol for Topology Discovery in Vehicular Ad Hoc Network", ANT/MobiWIS 2012: 525-534 (2012)

[18] Liren Zhang, Abderrahmane Lakas, Hesham ElSayed, Ezedin Barka, "Mobility analysis in vehicular ad hoc network (VANET)", J. Network and Computer Applications 36(3): 1050-1056 (2013)

[19] Hesham El-Sayed, Liren Zhang, Yaser E. Hawas, Hadeel El Kassabi, "A Histogram-Based Model for Road Traffic Characterization in VANET", IOV pp 42-55 ( 2014)

[20] Karim Emara, Wolfgang Woerndl, Johann Schlichter, "On evaluation of location privacy preserving schemes for VANET safety applications", Computer Communications, (2015). 